\title{
From checking the door to checking the app: assessment and treatment implications for OCD in the digital era Lior Carmi ${ }^{1,2}$, Joseph Zohar ${ }^{1}$, Oded Ben Arush ${ }^{3}$ and Sharon Morein-Zamir ${ }^{4}$
}

${ }^{1}$ Chaim Sheba Medical center, Ramat- Gan, Israel

${ }^{2}$ The Data Science Institution, The Inter Disciplinary Center, Herzliya, Israel

${ }^{3}$ Israeli Center for the Treatment of Obsessive-Compulsive Related Disorders, Modiin, Israel

${ }^{4}$ School of Psychology and Sport Science, Anglia Ruskin University, Cambridge, UK

Corresponding author: Dr. Lior Carmi, Email: Lior.Carmi@sheba.health.gov.il. Tel: +972502211969.

Address: Sheba Road 2, Sheba Medical Center, Ramat Gan, Israel 


\section{Abstract}

In the last twenty years technology is increasingly integrated into daily life. Daily interactions with smart devices have become routine with much of our lives taking place in a digital environment. It is therefore not surprising that the manifestations of psychiatric disorders including OCD have changed in recent years reflecting this reality. Clinicians should be aware of the potential impact of such changes when considering symptom presentation and diagnosis. 
Obsessive Compulsive Disorder (OCD) manifests globally with substantial consistency in prevalence, symptom clusters, and comorbidities (1). Although the major themes encompassed by OCD (e.g., contamination, checking, harm, and ordering) appear stable (2), the nature and content of specific obsessions and compulsions can change with cultural and social influences as well as historical context. For example, obsessions relating to AIDS appeared only in the late $20^{\text {th }}$ century (3). The biggest change in society in the last twenty years is arguably the penetrance of mobile phones into daily life, underlined by the increasing availability of the internet. With more than 5 billion projected smartphones users by 2025 (4), smartphones accompany individuals on an unprecedented scale, proving in some cases to be an extension of the self. Thus, the number of daily touches is estimated to top 2000 per day (5), with usage per day of up to 500 minutes (6), and $36-40 \%$ of smartphone owners using their smartphone within 5 min of going to sleep or of waking (7).

Given the sociological and cultural changes in the digital era, we propose that technology can influence how OCD is manifested, including the content of obsessions and the nature of compulsions. This is important as symptoms and signs of OCD in this new, rapidly evolving environment could at times be difficult to detect and fully comprehend.

\section{Obsessions and compulsions: manifesting digital symptoms}

Specific and elaborate inquiry is required to gain full understanding of OCD symptoms however they may present. Since technology manifests ubiquitously, and if anything, is set to become even more integral to patient lives, clinicians should ensure they actively assess for the presence of digital obsessions or compulsions. Considering that technology may influence the nature and form of OCD symptoms, clinical evaluation should routinely incorporate questions to detect digital-related expression in common symptom dimensions. These should be directed at possible interactions taking place in the virtual environment, such as with emails and social media ((8)). Additionally, interactions with 
ubiquitous technology such as smartphones, apps and intelligent personal assistant services (e.g., Amazon's Alexa, Apple's Siri) should also be probed. For example, compulsive checking may employ technological tools resulting in excessive list making on multiple dedicated apps, maladaptive use of webcams, or taking hundreds of pictures of windows and doors on one's smartphone camera. The immediacy and availability of such technology lends itself to maladaptive reassurance seeking and safety behaviours (9). The smartphone and apps within may also be subject to compulsive checking, for instance checking they open or close 'properly', or that messages were sent in the 'right' manner or time. Digital contamination obsessions can be expressed by rituals related to 'contaminated' websites. Similarly, digital ordering may revolve around one's messages, music files or any of the myriad of applications available. Hoarding, previously considered under OCD and now under the OC and Related Disorders category in the DSM-5 (American Psychiatric Association, 2013), may be expressed by impairing digital clutter and excessive churning, a common behaviour in hoarding disorder which may manifest in excessive time spent moving digital files on multiple storage devices (10). Not only may technology influence the content of OCD rituals but also interacting with technology may be dictated by manifestations of OCD, with avoidance of technology as a coping mechanism ultimately impairing functioning. The prominence of maladaptive interactions involving online social media is hardly unique to individuals with OCD but can posit specific challenges when repetitive obsessions or compulsions centre on potentially addictive elements such as rituals involving the number of Twitter followers (8). Clinicians should explicitly probe for digital symptoms as patient insight along with their perception of what is excessive and impairing may be limited. 


\section{Assessing digital obsessions and compulsions}

Symptom assessment and monitoring often relies on established and widely used tools

such as the Yale-Brown Obsessive Compulsive Scale Symptom Checklist (Goodman et al., 1989) or the Obsessive Compulsive Inventory (Foa et al., 2002). As these and other questionnaires were developed before the pervasiveness of smartphones and other digital technologies, we thus recommend routine augmentation of such tools. This could be implemented in practice by adding a 'digital layer' to each domain to reflect changes in the nature of OCD expression in the $21^{\text {st }}$ century.

Possible examples which could be probed include:

- Digital contamination, such as fears of contaminated websites or digital communications.

- Digital symmetry and ordering of electronic sources including games, emails, files, icons, and apps.

- Digital checking encompassing checking messages, 'likes', and emails and using smartphones to document ritual-related images or sounds.

- Digital compulsions encompassing repetitive interactions such as opening and closing apps and devices.

- Avoidance of virtual environments that result in impairment in everyday life and functioning.

Ultimately, tools measuring common OCD symptoms will need to be updated and revalidated to reflect the changing nature of how OCD can manifest. Awareness of the role of technology in communication, work and leisure practices between cultures may dictate further consideration. At the same time, there should be care to retain specificity to OCD symptoms per se, to avoid pathologising novel but ultimately normal clusters of behaviours and attitudes. Questionnaire and interview practices will likely have to continue to evolve as 
technological advances will undoubtedly shape the manifestation of symptoms in ways we cannot currently foresee.

Though not all patients with OCD will have digital compulsions and obsessions, anecdotal evidence has suggested to us that these are indeed present in some patients, resulting in significant distress and impairments. Ultimately, recognition of digital obsessions and compulsions will enable appropriate intervention and treatment. To conclude, we call attention to how technology is already changing the manifestation of neuro-psychiatric disorders such as OCD. Suitable and sensitive assessment tools for digital symptoms should be developed considering the evolution of digital technology.

\section{Declaration of interests' statement}

All authors declares no conflict of interests.

\section{References}

1 Weissman MM. Cross-national epidemiology of obsessive-compulsive disorder. CNS Spectrums. 1998;3(S1):6-9.

2 Leckman JF, Denys D, Simpson HB, et al. Obsessive-compulsive disorder: a review of the diagnostic criteria and possible subtypes and dimensional specifiers for DSM-V. Depression and anxiety. 2010;27(6):507-27.

3 Schechter JB, Myers MF, Solyom L. A case of obsessive-compulsive disorder related to AIDS: psychopharmacologic treatment. SAGE Publications Sage CA: Los Angeles, CA; 1991.

4 Miller $\mathrm{G}$. The smartphone psychology manifesto. Perspectives on psychological science. 2012;7(3):221-37.

5 Nelson P. We touch our phones 2,617 times a day, says study. Network World, July. 2016;7:353-75. 6 Falaki H, Mahajan R, Kandula S, Lymberopoulos D, Govindan R, Estrin D. Diversity in smartphone usage. Proceedings of the 8th international conference on Mobile systems, applications, and services; 2010: ACM; 2010. p. 179-94.

7 Montag C, Kannen C, Lachmann B, et al. The importance of analogue zeitgebers to reduce digital addictive tendencies in the 21st century. Addictive behaviors reports. 2015;2:23-7.

8 van Bennekom MJ, de Koning PP, Denys D. Social media and smartphone technology in the symptomatology of OCD. BMJ case reports. 2018;2018:bcr-2017-223662.

9 Carleton RN, Desgagné G, Krakauer R, Hong RY. Increasing intolerance of uncertainty over time: the potential influence of increasing connectivity. Cognitive behaviour therapy. 2019;48(2):121-36. 10 van Bennekom MJ, Blom RM, Vulink N, Denys D. A case of digital hoarding. Case Reports. 2015;2015:bcr2015210814. 